\title{
Retinol Modulates Site-specific Mobility of apo-Cellular Retinol-Binding Protein to Promote Ligand Binding
}

\author{
Tanja Mittag ${ }^{1+}$, Lorella Franzoni², Davide Cavazzini ${ }^{3}$, Brian Schaffhausen ${ }^{4}$, \\ Gian Luigi Rossi ${ }^{3}$ and Ulrich L. Günther ${ }^{* 5^{*}}$ \\ From J.W. Goethe University, Frankfurt, Center for Biomolecular Magnetic Resonance (BMRZ), Biocentre N230, \\ Marie-Curie-Str. 9, 60439 Frankfurt, Germany ${ }^{I},{ }^{2}$ Department of Experimental Medicine, Section of Chemistry \\ and Structural Biochemistry, University of Parma, 43100 Parma, Italy, ${ }^{3}$ Department of Biochemistry and \\ Molecular Biology, University of Parma, 43100 Parma, Italy, ${ }^{4}$ Department of Biochemistry, Tufts University, \\ School of Medicine, 136 Harrison Avenue, Boston, Massachusetts, ${ }^{5}$ CR UK Institute for Cancer Studies, \\ University of Birmingham, Vincent Drive, Edgbaston, Birmingham B15 2TT \\ *Address correspondence to: Ulrich Günther, CR UK Institute for Cancer Studies, University of Birmingham, \\ Vincent Drive, Edgbaston, Birmingham B15 2TT, UK, Tel. 0044 1214148361, Fax 0044 1214148357, email: \\ u.l.gunther@bham.ac.uk
}

\section{Materials and Methods}

\section{Protein titrations and line shape analysis}

Influence of methanol on apo-CRBP

$\mathrm{CD}_{3} \mathrm{OD}$ was added to apo-CRBP in steps of $5 \mu$. High resolution ${ }^{1} \mathrm{H} /{ }^{15} \mathrm{~N}-\mathrm{HSQC}$ spectra were recorded with the same parameters as for the titration with retinol. Line shapes were extracted for each residue and compared to the line shapes obtained for the titration with retinol. If the direction and extent of chemical shift changes were similar in both titrations, the effect was attributed to $\mathrm{CD}_{3} \mathrm{OD}$ rather than retinol.

\section{Influence of retinol on holo-CRBP}

The line shapes of a subset of residues revealed a third ligand dependent step with a high off-rate beginning at a retinol/protein ratio of 0.8 . To investigate the nature of this third step, excess retinol was removed from holoCRBP by passing the solution over a delipidating column. After a subsequent UV spectrum the solution still revealed total saturation of CRBP with retinol.

Further addition of retinol in $\mathrm{CD}_{3} \mathrm{OD}$ to holo-CRBP in similar steps as in the original titration resulted in identical small chemical shift changes as for low concentrations of retinol added to apo-CRBP.

Current address: Structural Biology and Biochemistry, Hospital for Sick Children, 555 University Avenue, Toronto, Ontario M5G 1X8, CANADA 


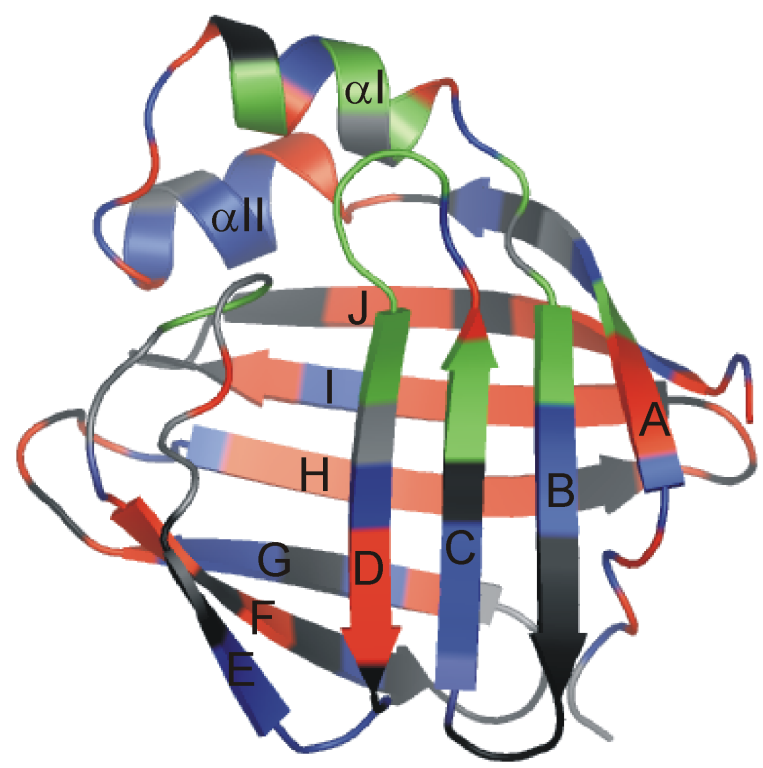

Figure 1: Ribbon diagram of apo-CRBP grouping residues according to their binding mechanism as revealed by line shape analysis. Black: no perturbation of the chemical shift upon addition of retinol; blue: one-step binding mechanism (eqn. 1) with signals in slow exchange; light blue: one-step binding mechanism (eqn. 1) with signals in fast exchange; red: two-step binding mechanism (eqn. 2); green: complex line shape with shoulders (eqn. 3); gray: no mechanism assignable due to missing assignment in one state or severe overlap. 